AEI-2010-053

\title{
Non-local gravity and the diffusion equation
}

\author{
Gianluca Calcagni \\ Max Planck Institute for Gravitational Physics (Albert Einstein Institute) \\ Am Mühlenberg 1, D-14476 Golm, Germany \\ E-mail: calcagni@aei.mpg.de \\ Giuseppe Nardelli \\ Dipartimento di Matematica e Fisica, Università Cattolica, \\ via Musei 41, 25121 Brescia, Italia \\ INFN Gruppo Collegato di Trento, Università di Trento, \\ 38100 Povo (Trento), Italia \\ E-mail: nardelli@dmf.unicatt.it
}

\begin{abstract}
We propose a non-local scalar-tensor model of gravity with pseudodifferential operators inspired by the effective action of $p$-adic string and string field theory on flat spacetime. An infinite number of derivatives act both on the metric and scalar field sector. The system is localized via the diffusion equation approach and its cosmology is studied. We find several exact dynamical solutions which are stationary in the diffusion flow. In particular, and contrary to standard general relativity, there exist de Sitter and power-law solutions also in an open universe, as well as solutions with sudden future singularities. Also, from the point of view of quantum field theory, spontaneous symmetry breaking can be naturally realized in the class of actions we consider.
\end{abstract}

Keywords: Cosmology of Theories beyond the SM, Quantum gravity phenomenology, String theory and cosmology. 


\section{Contents}

1. Introduction 1

2. Definition and dynamics 3

$2.1 p$-adic-like action 3

2.2 Equations of motion 6

3. $p$-adic-like cosmology

3.1 Friedmann equations 8

3.2 'Stationary' solutions with quadratic potential 8

3.3 General solutions with quadratic potential in conformal gravity 11

3.4 Asymptotic solutions and spontaneous symmetry breaking 18

4. String-like action and cosmology 20

5. Discussion 22

A. de Sitter and power-law solutions in standard local cosmology 24

\section{Introduction}

Many proposals for modified gravity have been invoked in the hope of finding new insights in the open issues of the standard cosmological model. Among them, theories with pseudo-differential operators have been favoured with particular attention. A reason is that non-local theories can have very different ultraviolet properties with respect to ordinary second- or higher-order actions (including popular Gauss-Bonnet extensions) and, hence, could play a role near the big bang and as spacetime effective formulations of non-perturbative quantum gravity. String field theory (SFT) is a concrete realization of this notion where pseudo-differential operators of the form

$$
e^{r_{*} \square}
$$

decorate the effective target action of the fields, where $r_{*}$ is a constant and $\square$ is the spacetime d'Alembertian. The imprint of non-local dynamics in the history of the early universe, or even as dark energy models, has motivated the study of cosmological models inspired by open SFT [1, 2, 3, 4, 5, 6, 7, 8, 9, 10, 11, 12, 13, 14, 15, 16, 17, 18, 19], the $p$-adic string [14, 15, 20, 21, 22, 23, 24], or other 
nonlocal effective actions featuring the operators (1.1] [25, 26] or inverse powers of the d'Alembertian [27, 28, 29, 30, 31, 32, 33. When non-locality is of the type (1.1), it can be conveniently manipulated with the diffusion equation approach, which has been developed and employed, in analytic and numerical fashion, under different formulations 110, 11, 12, 14, 15, 18, 23, 34, 35, 36, 37, 38, 39, 40, 41.

A rather common assumption in the literature of non-local fields in cosmology is that non-locality is confined only to one sector of the model, while the others are local. In the case of SFT-motivated actions, the non-local sector is matter (a scalar field) and gravity is local and with Einstein-Hilbert action:

$$
S=S_{\text {non-loc }}(\phi)+\frac{1}{2 \kappa^{2}} \int d^{D} x \sqrt{-g} R,
$$

where $D$ is the topological dimension of spacetime, $g$ is the determinant of the metric $g_{\mu \nu}, \mu=0, \ldots, D-1, \kappa^{2}=8 \pi G$ is Newton's constant and $R$ is the Ricci curvature scalar. This Ansatz has been dictated mainly by the urgency of understanding, in the broadest sense, (i) the dynamics of the yet-unclear non-local scalar field theories, (ii) the combined effect of curvature and nonlocality, and (iii) its possible consequences for phenomenology, in particular in relation with cosmology (inflation, dark energy) and the modification of flat open SFT solutions (can cosmological friction damp the wild oscillations of the OSFT solution with marginal deformations? [4, 14, 16, 18, 35).

Now that robust analytical and numerical methods have been established to solve non-local equations of motion, it would be highly desirable to address the conceptual inconsistency subjacent to Eq. (1.2). Not only would we like to define a model with non-locality implemented in all sectors (and reproducing standard general relativity in the limit of weak non-locality), but we want also to find non-trivial cosmological solutions. Such is the twofold objective of this paper.

The problem of non-local gravity can be faced under three independent perspectives, one motivated by string field theory, one purely phenomenological and another a hybrid approach. In the first case, the Einstein-Hilbert action in eq. (1.2) is introduced by hand as an educated guess on 'how the effective SFT action of tachyon might look like in the presence of gravity'. The latter is minimally coupled with a tachyontype or $p$-adic scalar field whose action is dictated or inspired by concrete Minkowski calculations. Obviously, a fully consistent effective tachyonic action should be derived from first principles in all its sectors. As far as gravity is concerned, the natural framework is closed SFT [42, 43, 44, 45, 46, 47, 48, 49, 50, 51, 52, 53, 54, 55, 56], which features the same non-local operator (1.1) of open SFT. The subject is rather intricate and, unfortunately, effective gravitational non-local actions are known only at linear level [45, 56]. ${ }^{1}$

\footnotetext{
${ }^{1}$ On the other hand, the local low-energy effective field theory of the closed string tachyondilaton-graviton system is well understood also in its cosmological properties [57, 58].
} 
Instead of facing the rigors of closed SFT some toy models have been considered, in particular the open-closed $p$-adic tachyonic action [59, 60, 61] and a closed SFTinspired tachyon-tachyon model [62]. However, the graviton is not included, thus leaving the possibility to consider phenomenological actions where the matter sector be as close as possible to SFT or the $p$-adic string, while a non-local gravitational sector is built from reasonable requirements (mainly, that it contains the same type of pseudo-differential operators as the matter sector). Here we shall follow the third path.

Non-local gravity sectors have been constructed with inverse powers of the operator 27, 28, 29, 30, 31, 32, 33] or more general kinetic functions [26], while keeping matter local. In 25] a non-local total action has been proposed with nonminimal coupling between gravity and a scalar field, but the dynamical analysis therein does not go beyond cosmological solutions when the matter sector is switched off.

In section 2 we adopt the diffusion equation method to infer the form of a solvable scalar-tensor non-local action with pseudo-differential operators of exponential type, eq. (1.1). This approach is chosen by virtue of its non-perturbative character, which does not require to truncate the theory in order to find solutions, exact or asymptotic. Exact non-vacuum solutions of the equations of motion of a $p$-adic like system will be found in section 3 for cosmological backgrounds; their classical stability is checked. The exact solutions are stationary along the diffusion flow (i.e., the diffusion equation is trivially satisfied) but the scalar and Hubble profiles as well as their dynamics are non-trivial. Notably, there exist de Sitter and power-law solutions with negative intrinsic curvature (section 3.2), as well as a most general class of explicit solutions for actions with conformal operators (section 3.3).

We make some general remarks on non-stationary asymptotic solutions in section 3.4, showing that, for natural choices of the potential, the system realizes spontaneous symmetry breaking. In section 1 the analysis is extended to another action with kinetic operator similar to the one of the SFT tachyon; de Sitter and power-law exact solutions are found. Section 5 is devoted to discussion. In the appendix we recall flat and curved de Sitter and power-law solutions in standard general relativity.

\section{Definition and dynamics}

\section{$2.1 p$-adic-like action}

Let us begin for simplicity with the $p$-adic action in Minkowski spacetime,

$$
S=\int d^{D} x\left[\frac{1}{2} \phi e^{r_{*} \square} \phi-V(\phi)\right]
$$

where $V$ is the field potential and we used dimensionless units (a mass factor $M^{-2}$ is hidden in $\square$, where $M$ is the characteristic energy scale of the system). In the 
diffusion equation method [38, 41], one introduces an auxiliary direction $r$ along which the scalar field diffuses according to the equation

$$
\left(\square+\partial_{r}\right) \phi(r, x)=0
$$

Since

$$
\left[\square, \partial_{r}\right]=0,
$$

the action of the operators (1.1) is simply a translation along $r$ :

$$
e^{r_{*} \square} \phi(r, x)=e^{-r_{*} \partial_{r}} \phi(r, x)=\phi\left(r-r_{*}, x\right) .
$$

As a consequence, the system becomes localized in spacetime, it has a well-defined Cauchy problem 38 and the equation of motion can be solved by choosing an appropriate initial field configuration $\phi(0, x)$. Examples are the exact solution for the $p$-adic string 23] and approximate solutions for open string and superstring field theory 37, 40, 41].

When introducing a non-trivial metric $g_{\mu \nu}$, we have to include also a non-local action for it. As far as the diffusion method is concerned, so far this has not been done, and discussions have been limited to systems of the form (1.2) [10] or with more general local gravitational actions 18. The reason was mainly technical. Suppose to decorate the gravitational action with the exponential operators (1.1). If we hope to solve the system analytically or semi-analytically, it is reasonable to expect that a metric-derived field obey the diffusion equation. For instance, this could be the metric itself $g_{\mu \nu}(r, x)$ or one of the Riemann invariants $R(g) \equiv R_{\mu \nu \ldots}(r, x)$; different choices are physically inequivalent. Whatever the choice, the fundamental commutation property (2.3) is no longer valid, because now the d'Alembertian is coordinate dependent and the diffusion equation for the metric or Riemann invariant $R(g)$ is essentially non-linear:

$$
\nabla_{\sigma}(g) \nabla^{\sigma}(g) R(g)=-\partial_{r} R(g)
$$

where $\nabla_{\sigma}$ is the covariant derivative. Accordingly,

$$
\begin{aligned}
\square^{2} R(g) & =-\square \partial_{r} R(g) \neq-\partial_{r} \square R(g) \\
& \neq \partial_{r}^{2} R(g) \\
& \cdots \\
\square^{n} R(g) & \neq(-1)^{n} \partial_{r}^{n} R(g),
\end{aligned}
$$

and the translation property (2.4) breaks down for $R(g)$.

However, our main goal is not really to impose the diffusion equation in all sectors, but rather (i) to include an infinite number of derivatives of the metric field for consistency with the matter sector, and (ii) do it in while preserving the translation 
property wherever and whenever required. Therefore, we can look towards another direction: namely, give up diffusion in metric fields,

$$
\partial_{r} R(g)=0,
$$

and modify the diffusion equation with an extra curvature term. Here we take for simplicity a term depending only on the Ricci scalar:

$$
\left[\square+f(R)+\partial_{r}\right] \phi=0,
$$

where $f$ is a function. Equation (2.1) is modified as

$$
S=\int d^{D} x \sqrt{-g}\left[\frac{1}{2} \phi e^{r_{*}[\square+f(R)]} \phi-V(\phi)\right] .
$$

The role of the $f(R)$ term is to realize non-locality in the gravity sector: an infinite number of derivatives act on metric fields via the Baker-Campbell-Hausdorff formula. This way the same exponential operator act on both sectors and one can still solve the equations of motion non-perturbatively and analytically, either exactly or approximately.

Equation (2.8) is the total action for our scalar-tensor model. The scalar sector diffuses while the metric sector does not, but both sectors are non-local as desired. The resulting equations of motion can be localized ( $\phi$ translates, $e^{r_{*}[\square+f(R)]} \phi(r, x)=$ $\left.\phi\left(r-r_{*}, x\right)\right)$ and solved for a given fixed metric. We denote as

$$
\phi(1, x)=\phi(x)
$$

the scalar field in the action, that is, $\phi$ evaluated at the end of the diffusion flow.

Equation (2.8) has well-defined limits as a pure scalar field or gravitational theory. Let $f(0)=0$. In the Minkowski limit, eq. (2.8) reduces to eq. (2.1). On the other hand, when $\phi$ relaxes to a constant $\phi_{0}$ (local minimum of the potential, $\left.V_{0} \equiv V\left(\phi_{0}\right) \equiv \phi_{0}^{2} \Lambda\right)$ one has, up to an overall constant,

$$
S \sim \int d^{D} x \sqrt{-g}\left\{e^{r_{*}[\square+f(R)]}-2 \Lambda\right\},
$$

which becomes an ordinary $f(R)$ theory in the small $r_{*}$ limit. In the same limit but keeping $\phi$ dynamical,

$$
S \sim \int d^{D} x \sqrt{-g}\left\{\frac{1}{2} \phi\left[r_{*} \square+r_{*} f(R)\right] \phi-V(\phi)\right\} .
$$

Later on we will find solutions of the full non-local action for the linear case $f(R)=$ $\alpha R$, where $\alpha$ is a constant which will be often set to a negative value. To recover general relativity in eq. (2.11), it will be sufficient to choose $r_{*}<0$. Then, the kinetic term for the scalar field has the wrong sign but this does not correspond to a ghost, 
since eq. (2.11) is only an effective action. The full action (2.8) is not plagued by any such instability, and in fact there are no physical poles at all. Notice also that restoring mass units, factorizing $R / M^{2}$ and looking at the weak-non-locality limit (2.11), one has

$$
|\alpha|=\frac{M^{2}}{8 \pi G}=\left(\frac{M}{M_{\mathrm{Pl}}}\right)^{2}<1,
$$

where $M_{\mathrm{Pl}}$ is the reduced Planck mass. The stable solutions we shall find nicely satisfy this condition; therefore, their energy scale is naturally below the Planck scale. $^{2}$

Although the motivations underlying our Ansatz (2.8) differ from those of [25], we eventually got a very similar result. This is not completely unexpected because both the diffusion equation and the ultraviolet properties invoked in the construction of 25] (absence of ghosts and asymptotic freedom) rely on eq. (1.1). The diffusion equation encodes some of the gauge symmetries of SFT at spacetime level [41] and, as such, gives rise to rather rigid physical properties.

\subsection{Equations of motion}

To find the equations of motion we need the variations

$$
\begin{aligned}
\delta \sqrt{-g} & =-\frac{1}{2} g_{\mu \nu} \sqrt{-g} \delta g^{\mu \nu}, \\
\delta R & =\left(R_{\mu \nu}+g_{\mu \nu} \square-\nabla_{\mu} \nabla_{\nu}\right) \delta g^{\mu \nu},
\end{aligned}
$$

where $\nabla_{\nu} V_{\mu} \equiv \partial_{\nu} V_{\mu}-\Gamma_{\mu \nu}^{\sigma} V_{\sigma}$ is the covariant derivative of a vector $V_{\mu}$ and the curved d'Alembertian on a scalar $\phi$ is

$$
\square \phi=\frac{1}{\sqrt{-g}} \partial^{\mu}\left(\sqrt{-g} \partial_{\mu} \phi\right)
$$

We will make use of the operator identity 63

$$
\delta e^{r_{*} X}=\int_{0}^{r_{*}} d s e^{s X}(\delta X) e^{\left(r_{*}-s\right) X},
$$

for a (differential) operator $X$.

Also, for two scalars $\Phi_{1}$ and $\Phi_{2}(\rightarrow$ indicates integration by parts),

$$
\begin{aligned}
\sqrt{-g} \Phi_{1} \frac{\delta \square}{\delta g^{\mu \nu}} \Phi_{2} & =\Phi_{1} \partial_{\mu}\left(\sqrt{-g} \partial_{\nu} \Phi_{2}\right)-\frac{1}{2} \sqrt{-g} \Phi_{1}\left(\partial^{\sigma} g_{\mu \nu}\right) \partial_{\sigma} \Phi_{2} \\
& \rightarrow \sqrt{-g}\left[\frac{1}{2} g_{\mu \nu}\left(\Phi_{1} \square \Phi_{2}+\partial_{\sigma} \Phi_{1} \partial^{\sigma} \Phi_{2}\right)-\left(\partial_{\mu} \Phi_{1}\right)\left(\partial_{\nu} \Phi_{2}\right)\right]
\end{aligned}
$$

\footnotetext{
${ }^{2}$ The only exception is eq. (3.23) for $p$ near 0 or $2 / D$. However, solutions with these fine-tuned $p$ 's do not inflate.
} 
and

$$
\begin{aligned}
\sqrt{-g} \Phi_{1} \Phi_{2} \frac{\delta f(R)}{\delta g^{\mu \nu}} & =\sqrt{-g} \Phi_{1} \Phi_{2} f^{\prime}(R) \frac{\delta R}{\delta g^{\mu \nu}} \\
& \rightarrow \sqrt{-g}\left\{\Phi_{1} \Phi_{2} f^{\prime}(R) R_{\mu \nu}+\left(g_{\mu \nu} \square-\nabla_{\mu} \nabla_{\nu}\right)\left[\Phi_{1} \Phi_{2} f^{\prime}(R)\right]\right\}
\end{aligned}
$$

where we have discarded boundary terms.

The scalar equation of motion $\delta S / \delta \phi=0$ is

$$
\phi\left(1-r_{*}, x\right)=V^{\prime}[\phi(1, x)] .
$$

The Einstein equations are (coordinate dependence of the fields implicit)

$$
\begin{aligned}
0= & \frac{2}{\sqrt{-g}} \frac{\delta S}{\delta g^{\mu \nu}} \\
= & -g_{\mu \nu}\left\{\frac{1}{2} \phi(1) \phi\left(1-r_{*}\right)-V[\phi(1)]\right\} \\
& +\frac{1}{\sqrt{-g}} \int_{0}^{r_{*}} d s \int d^{D} x \sqrt{-g} \phi(1-s)\left[\frac{\delta \square}{\delta g^{\mu \nu}}+\frac{\delta f(R)}{\delta g^{\mu \nu}}\right] \phi\left(1-r_{*}+s\right) \\
= & -g_{\mu \nu}\left\{\frac{1}{2} \phi(1) \phi\left(1-r_{*}\right)-V[\phi(1)]\right\}+\int_{0}^{r_{*}} d s \Sigma_{\mu \nu}(s) \\
\Sigma_{\mu \nu}(s)= & \frac{1}{2} g_{\mu \nu}\left[\phi(1-s) \square \phi\left(1-r_{*}+s\right)+\partial_{\sigma} \phi(1-s) \partial^{\sigma} \phi\left(1-r_{*}+s\right)\right] \\
& -\partial_{\mu} \phi(1-s) \partial_{\nu} \phi\left(1-r_{*}+s\right)+\phi(1-s) \phi\left(1-r_{*}+s\right) f^{\prime}(R) R_{\mu \nu} \\
& +\left(g_{\mu \nu} \square-\nabla_{\mu} \nabla_{\nu}\right)\left[\phi(1-s) \phi\left(1-r_{*}+s\right) f^{\prime}(R)\right] .
\end{aligned}
$$

Taking the trace,

$$
\begin{aligned}
0= & -D\left\{\frac{1}{2} \phi(1) \phi\left(1-r_{*}\right)-V[\phi(1)]\right\}+\int_{0}^{r_{*}} d s \Sigma(s), \\
\Sigma(s)= & \frac{D}{2} \phi(1-s) \square \phi\left(1-r_{*}+s\right)+\left(\frac{D}{2}-1\right) \partial_{\sigma} \phi(1-s) \partial^{\sigma} \phi\left(1-r_{*}+s\right) \\
& +\phi(1-s) \phi\left(1-r_{*}+s\right) f^{\prime}(R) R+(D-1) \square\left[\phi(1-s) \phi\left(1-r_{*}+s\right) f^{\prime}(R)\right] .
\end{aligned}
$$

\section{3. $p$-adic-like cosmology}

A natural background whereon to study the model is Friedmann-Robertson-Walker (FRW), defined by the line element

$$
d s^{2}=g_{\mu \nu} d x^{\mu} d x^{\nu}=-d t^{2}+a(t)^{2} \tilde{g}_{i j} d x^{i} d x^{j},
$$

where $t$ is synchronous time, $a(t)$ is the scale factor and

$$
\tilde{g}_{i j} d x^{i} d x^{j}=\frac{d \varrho^{2}}{1-\mathrm{K} \varrho^{2}}+\varrho^{2} d \Omega_{D-2}^{2}
$$


is the line element of the maximally symmetric $(D-1)$-dimensional space $\tilde{\Sigma}$ of constant sectional curvature $\mathrm{K}$ (equal to -1 for an open universe, 0 for a flat universe and +1 for a closed universe with radius $a$ ).

\subsection{Friedmann equations}

On an FRW background,

$$
\begin{aligned}
& R_{00}=-(D-1)\left(H^{2}+\dot{H}\right), \\
& R_{i j}=\tilde{R} g_{i j} \equiv\left[\frac{2 \mathrm{~K}}{a^{2}}+(D-1) H^{2}+\dot{H}\right] g_{i j}, \\
& R=(D-1)\left(\frac{2 \mathrm{~K}}{a^{2}}+D H^{2}+2 \dot{H}\right),
\end{aligned}
$$

where

$$
H \equiv \frac{\dot{a}}{a}
$$

is the Hubble parameter. The 00 component of Einstein's equations is

$$
\begin{aligned}
0= & \frac{1}{2} \phi(1) \phi\left(1-r_{*}\right)-V[\phi(1)]+\int_{0}^{r_{*}} d s \Sigma_{00}(s), \\
\Sigma_{00}(s)= & -\frac{1}{2} \phi(1-s) \square \phi\left(1-r_{*}+s\right)-\frac{1}{2} \dot{\phi}(1-s) \dot{\phi}\left(1-r_{*}+s\right) \\
& +\phi(1-s) \phi\left(1-r_{*}+s\right) f^{\prime}(R) R_{00} \\
& +(D-1) H \partial_{t}\left[\phi(1-s) \phi\left(1-r_{*}+s\right) f^{\prime}(R)\right] .
\end{aligned}
$$

We specialize to the case

$$
f(R)=\alpha R
$$

where $\alpha$ is a dimensionless constant. In the Friedmann equations (3.8) and (2.23) one has

$$
\begin{aligned}
\Sigma_{00}(s)= & -\frac{1}{2} \phi(1-s) \square \phi\left(1-r_{*}+s\right)-\frac{1}{2} \dot{\phi}(1-s) \dot{\phi}\left(1-r_{*}+s\right) \\
& -\alpha(D-1)\left(H^{2}+\dot{H}\right) \phi(1-s) \phi\left(1-r_{*}+s\right) \\
& +\alpha(D-1) H \partial_{t}\left[\phi(1-s) \phi\left(1-r_{*}+s\right)\right]
\end{aligned}
$$

and

$$
\begin{aligned}
\Sigma(s)= & \frac{D}{2} \phi(1-s) \square \phi\left(1-r_{*}+s\right)-\left(\frac{D}{2}-1\right) \dot{\phi}(1-s) \dot{\phi}\left(1-r_{*}+s\right) \\
& +\alpha \phi(1-s) \phi\left(1-r_{*}+s\right) R+\alpha(D-1) \square\left[\phi(1-s) \phi\left(1-r_{*}+s\right)\right] .
\end{aligned}
$$

\section{2 'Stationary' solutions with quadratic potential}

Although the diffusion method allows one to find asymptotic solutions for any given

background, it is not necessary to resort to all its machinery in order to get some 
interesting results. In particular, one can look for simple solutions which are trivial along the diffusion flow, i.e.,

$$
\phi(r, t)=e^{\beta r} \varphi(t)
$$

where $\beta$ is a constant and $\varphi$ is a stationary solution of the diffusion equation. ${ }^{3}$ Since $\beta$ only changes the normalization of the field and its potential, we can set $\beta=0$ without loss of generality:

$$
(\square+\alpha R) \varphi=0 .
$$

Later on we will actually see that most of the solutions do require $\beta=0$.

Although the $r$-dependence is trivial, eq. (3.13) contains a class of full-fledged dynamical solutions which are non-trivial in time and, from eq. (2.19), have

$$
V=\frac{\varphi^{2}}{2}
$$

The sign of the effective potential depends on the sign of $r_{*} \alpha R$ :

$$
W(\varphi)=\left(1-e^{r_{*} \alpha R}\right) \frac{\varphi^{2}}{2}
$$

Unless indicated otherwise, we will choose the sign of $r_{*}$ so that $W$ is bounded from below. Since the interaction is quadratic, we regard this case as perturbative in a quantum field theory sense, although it is still fully non-local.

The Einstein equations on stationary solutions are very simple. For any background, $\Sigma_{\mu \nu}=0$ :

$$
0=\left(\frac{1}{2}+2 \alpha\right) g_{\mu \nu}\left(\varphi \square \varphi-\dot{\varphi}^{2}\right)-(1+2 \alpha) \partial_{\mu} \varphi \partial_{\nu} \varphi+\alpha \varphi^{2} R_{\mu \nu}-2 \alpha \varphi \nabla_{\mu} \nabla_{\nu} \varphi,
$$

where we used eqs. (2.20), (3.14) and $\square\left(\varphi^{2}\right)=2 \varphi \square \varphi-2 \dot{\varphi}^{2}$. After using eq. (3.13), the Friedmann (00 and trace) equations become

$$
\begin{aligned}
& 0=\left[\frac{\mathrm{K}}{a^{2}}+\left(\frac{D}{2}-1\right) H^{2}\right] \varphi^{2}-\frac{1}{2 \alpha(D-1)} \dot{\varphi}^{2}+2 H \varphi \dot{\varphi}, \\
& 0=[4 \alpha(D-1)+D-2]\left(\dot{\varphi}^{2}+\alpha R \varphi^{2}\right) .
\end{aligned}
$$

The second equation is automatically satisfied for

$$
\alpha=-\alpha_{*} \equiv-\frac{1}{4} \frac{D-2}{D-1} .
$$

For the time being we assume $\alpha \neq \alpha_{*}$ and present solutions to the Einstein's equations with non-trivial $\varphi(t)$ and $a(t)$ profiles.

We start with de Sitter metric,

$$
a(t)=e^{H t}, \quad H=\text { const. }
$$

\footnotetext{
${ }^{3}$ The 'Wick-rotated' diffusion equation $(r \rightarrow i r)$ is a Schrödinger equation, so $\beta$ is the analogue of the energy eigenvalue of stationary solutions.
} 
For a flat background $\mathrm{K}=0$, one finds

$$
\varphi(t)=e^{-\frac{1}{2}(D-1) H t}, \quad \alpha=-\frac{D-1}{4 D} .
$$

The scalar field rolls down its potential towards its minimum, reaching it asymptotically. This feature is similar to what found in [18] (where, however, the Einstein equations were not solved) and is typical of non-local models where the cosmological friction is enhanced by non-local operators. This is determined by the form of the non-local equations of motion, and it happens even in this case where the non-local operators are trivialized on stationary solutions.

Another class of solutions is power law:

$$
\begin{aligned}
& a(t)=t^{p}, \\
& \varphi(t)=t^{q} .
\end{aligned}
$$

Inflation $(\ddot{a}>0)$ happens when $p>1$. When $\mathrm{K}=0$, the solution is

$$
\alpha=-\frac{(D p-p-1)^{2}}{4(D-1)(D p-2) p}, \quad p \neq 1, \quad q=-\frac{1}{2}(D p-p-1) .
$$

In four dimensions and for $p>1 / 3, q<0$ and the field rolls down towards the minimum.

We now check the classical stability of these solutions in synchronous time formalism. ${ }^{4}$ A background solution $(H(t), \varphi(t))$ is perturbed homogeneously,

$$
H(t) \rightarrow H(t)+\delta H(t), \quad \varphi(t) \rightarrow \varphi(t)+\delta \varphi(t)
$$

and the equations of motion (3.13), (3.17) and (3.18) are linearized (the scalar equation of motion is an identity). The system can be written in a matrix form:

$$
\delta \dot{X}=M \delta X
$$

where

$$
\delta X \equiv\left(\begin{array}{c}
\delta H \\
\delta \varphi
\end{array}\right)
$$

\footnotetext{
${ }^{4}$ For an introduction to phase space analysis, see [64]. Here it is worth mentioning a caveat about the choice of clocks. In general, time $t$ is an unphysical parameter and one has to choose an internal physical clock. This can be one of the matter fields in the total action, e.g., a scalar field or a barotropic fluid. Failure to do so can sometimes lead to inconsistencies in the stability analysis. The reason 65] is that perturbations in synchronous time discriminate between trajectories differing only by a shift in time, which are actually physically equivalent and should be identified. On the other hand, in the presence of an internal clock time shifts can be physically distinguished. In the analysis below, this turns out not to be the case. One can convince oneself by noticing that an extra dust component in the system would leave the perturbed equations unchanged.
} 
and the entries $m_{i j}=(M)_{i j}$ of the $2 \times 2$ matrix $M$ are calculated on the background solution. The characteristic equation

$$
\operatorname{det}(M-\lambda \mathbb{I})=0
$$

determines the eigenvalues $\lambda$ and a solution is stable provided $\operatorname{Re}(\lambda) \leq 0$. In general, the eigenvalues are time dependent, in which case they are interpreted as evaluated at a given time $t$ 66.

For all the solutions above $m_{12}=0$, so that $\lambda_{1}=m_{11}$ and $\lambda_{2}=m_{22}$. For the de Sitter solution (3.21),

$$
\lambda_{1}=0, \quad \lambda_{2}=-\frac{1}{2}(D-1) H<0,
$$

thus implying stability. For the power-law solution (3.23) $(t>0)$,

$$
\lambda_{1}=-\frac{2}{t}, \quad \lambda_{2}=-\frac{(D-1) p+1}{2 t},
$$

the latter being negative when

$$
p>-\frac{1}{D-1}
$$

which is true for an expanding universe $(p>0)$.

\subsection{General solutions with quadratic potential in conformal gravity}

The flat de Sitter solution can be generalized straightforwardly to any $f(R)$ theory. In fact, it is sufficient to make the replacement

$$
\alpha \rightarrow \alpha_{\mathrm{eff}} \equiv f^{\prime}\left(R_{\mathrm{dS}}\right)
$$

in eq. (3.21), where $R_{\mathrm{dS}}=D(D-1) H^{2}$. Depending on the form of $f$, the value of the Hubble constant is determined by $\alpha_{\text {eff }}$.

Another generalization is based on the fact that the most general solution with $\alpha=-\alpha_{*}$ can be found for any FRW background via the following shortcut.

The critical value eq. (3.19) is well known in conformal gravity models [67]. In four dimensions, $\alpha=-1 / 6$. Consider a metric $g_{\mu \nu}$ and the conformal transformation

$$
\begin{aligned}
\bar{g}_{\mu \nu} & \equiv \Omega^{2} g_{\mu \nu}, \\
\bar{\phi} & \equiv \Omega^{1-\frac{D}{2}} \phi,
\end{aligned}
$$

for some $r$-independent $\Omega=\Omega(x)$. The Christoffel symbols transform as

$$
\Gamma_{\mu \nu}^{\lambda}=\bar{\Gamma}_{\mu \nu}^{\lambda}-\left[2 \delta_{(\mu}^{\lambda} \mathcal{O}_{\nu)}-\bar{g}_{\mu \nu} \mathcal{O}^{\lambda}\right]
$$


where

$$
\mathcal{O}_{\mu} \equiv \bar{\nabla}_{\mu} \ln \Omega, \quad \mathcal{O} \equiv \bar{\nabla}^{\mu} \mathcal{O}_{\mu}=\bar{\square} \ln \Omega
$$

Noting that

$$
\begin{aligned}
& \Omega^{-2} R=\bar{R}+2(D-1) \mathcal{O}-(D-1)(D-2) \mathcal{O}_{\mu} \mathcal{O}^{\mu}, \\
& \Omega^{-2} \square=\square-(D-2) \mathcal{O}^{\mu} \bar{\nabla}_{\mu},
\end{aligned}
$$

one can see that the combination $(\square+\alpha R) \phi$ has a well-defined conformal weight only if $\alpha=-\alpha_{*}$ :

$$
\left(\square-\alpha_{*} R\right) \phi=\Omega^{2}\left(\bar{\square}-\alpha_{*} \bar{R}\right) \bar{\phi} .
$$

On a background where $\bar{R}=R_{0}=$ const, the diffusion equation is equivalent to

$$
\Omega^{2}\left(\bar{\square}-\alpha_{*} R_{0}+\Omega^{\frac{D}{2}-3} \partial_{r}\right) \bar{\phi}=0 \text {. }
$$

We specialize now to FRW backgrounds, where the metric can be written in conformal time

$$
\tau \equiv \int \frac{d t}{a(t)}
$$

so that $\Omega=a^{-1}, \square=-\partial_{\tau}^{2}$ and $R_{0}=2(D-1) \mathrm{K}$. For stationary solutions, the diffusion equation reduces to eq. (3.38):

$$
\bar{\varphi}^{\prime \prime}+\operatorname{sgn}(\mathrm{K}) \gamma^{2} \bar{\varphi}=0,
$$

where

$$
\gamma^{2} \equiv\left(\frac{D}{2}-1\right)|\mathrm{K}|
$$

and primes denote derivatives with respect to $\tau$. Just solving this equation, one obtains a very wide class of solutions from eq. (3.33),

$$
\varphi(\tau)=[a(\tau)]^{1-\frac{D}{2}} \bar{\varphi}(\tau) .
$$

In fact, the whole dynamics reduces to one equation, encoding both eq. (3.40) and a constraint on $\bar{\varphi}^{\prime}$ and the curvature. To show this, instead of the trace equation (3.18) one takes the $i i$-component equation $\Sigma_{i i}=0$,

$$
0=-\left(\frac{1}{2}+2 \alpha\right)\left(\dot{\varphi}^{2}+\alpha R \varphi^{2}\right)+\alpha \varphi^{2} \tilde{R}+2 \alpha H \varphi \dot{\varphi}
$$

Replacing eq. (3.42) in eqs. (3.17) and (3.43) with $\alpha=-\alpha_{*}$, one obtains

$$
\left(\bar{\varphi}^{\prime}\right)^{2}+\operatorname{sgn}(\mathrm{K}) \gamma^{2} \bar{\varphi}^{2}=0
$$

which can be solved only if $\mathrm{K}=0$ or $\mathrm{K}=-1$. There are no closed universe solutions. Differentiation of eq. (3.44) in conformal time yields eq. (3.40), so for the critical value (3.19) the stationary problem is drastically simplified. This is possible only for 
stationary solutions, where geometry is factorized out of the equations of motion. Let us consider a few concrete examples.

Flat universe $(\mathrm{K}=0)$. The $\bar{g}$ frame is Minkowski and $\bar{\varphi}=1$ is solution of the free equation $\bar{\varphi}^{\prime \prime}=0 .^{5}$ Then, the general solution is

$$
\varphi(\tau)=[a(\tau)]^{1-\frac{D}{2}} .
$$

For the de Sitter background (3.20) in synchronous time,

$$
\varphi(t)=e^{-\left(\frac{D}{2}-1\right) H t},
$$

and the evolution of the scalar field is qualitatively the same as for eq. (3.21).

For the power-law profile (3.22), the solution has

$$
q=-\left(\frac{D}{2}-1\right) p
$$

Since the algorithm given by eqs. (3.40) and (3.42) is valid for any choice of the scale factor, there is an infinite set of solutions without big bang singularity. A $D=4$ bouncing solution with zero intrinsic curvature is $\left(r_{*}>0\right)$

$$
\begin{aligned}
& a(t)=\frac{1}{\varphi(t)}=\cosh t \\
& H=\tanh t, \quad R=6\left[1+(\tanh t)^{2}\right] .
\end{aligned}
$$

In the contracting phase the scalar field rolls from its minimum up to a maximum value. At the inversion point the universe bounces and starts expanding, while $\varphi$ roll back towards the global minimum. This solution is the scalar-tensor analogue, in the sense of eq. (2.8), of the bouncing solution of [25]. The fact that the scale factor $a$ is non-singular, however, is to be ascribed more to the choice of a conformal operator rather than to the good ultraviolet properties of non-local theories.

Another bouncing profile is the super-accelerating cosmology

$$
a(t)=\exp \left(\frac{1}{2} H_{0} t^{2}\right), \quad H=H_{0} t
$$

which is plotted in figure 11 for $D=4$. The scalar field evolves from the global minimum up to the inversion point $\varphi=1$ at the bounce. Then, cosmological friction drags it back to the minimum.

Open universe $(\mathrm{K}=-1)$. In the open case, eq. (3.40) is solved by exponentials and there are two general dynamical solutions which read

$$
\varphi_{ \pm}(\tau)=[a(\tau)]^{-\gamma^{2}} e^{\mp \gamma \tau}
$$

\footnotetext{
${ }^{5}$ Also $\bar{\varphi}=\tau$ solves the diffusion equation, but it does not solve eq. (3.44).
} 


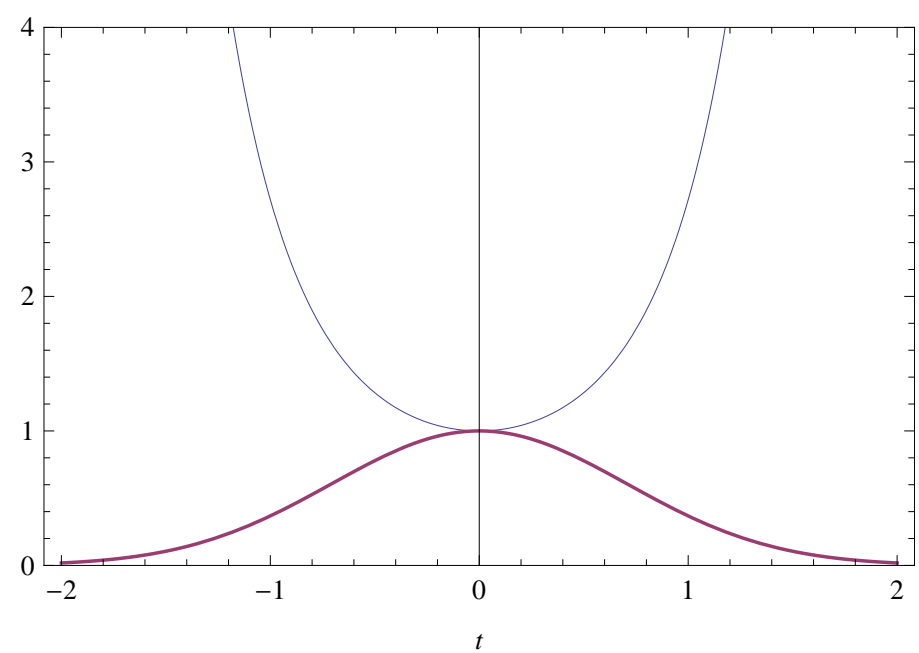

Figure 1: Bouncing $\mathrm{K}=0$ solution $(3.49)$ with $D=4$ and $H_{0}=2$. Thin line: scale factor $a(t)$; thick line: scalar profile $\varphi(t)=1 / a(t)$.

Therefore, and somehow surprisingly, there are also two de Sitter solutions for an open universe:

$$
\varphi_{ \pm}(t)=e^{-\gamma^{2} H t} \exp \left( \pm \frac{\gamma}{H} e^{-H t}\right)
$$

where we used $\tau=-e^{-H t} / H$. The profile $\varphi_{+}$has the same features as the flat solutions and rolls towards the global minimum. The solution $\varphi_{-}$climbs from the minimum up to some maximum value, then rolls back down (figure 2).

For the power-law profiles (3.22), when $p=1$ (linear scale factor) the solutions have

$$
q_{ \pm}=-\sqrt{\frac{D}{2}-1}\left(\sqrt{\frac{D}{2}-1} \pm 1\right) .
$$

In four dimensions, only the positive root is non-trivial, $q=-2$. For arbitrary $p$, the solutions are

$$
\varphi_{ \pm}(t)=t^{-\gamma^{2} p} e^{ \pm \frac{\gamma}{p-1} t^{1-p}}
$$

The typical plot of, say, $\varphi_{-}$is shown in figure 3 and is similar to the previous one. For comparison, the exact de Sitter and power-law solutions in standard general relativity are reported in the appendix.

For the bouncing solution eq. (3.49), the conformal time is

$$
\tau=\sqrt{\frac{\pi}{2 H_{0}}} \operatorname{erf}\left(\sqrt{\frac{H_{0}}{2}} t\right),
$$

thus leading to the asymmetric lump $\varphi_{-}(t)$ shown in figure 4 . The evolution is similar to the flat case, except that now open geometry helps the scalar field to climb up to a higher inversion point before being dragged back by cosmic super-friction. The lump 

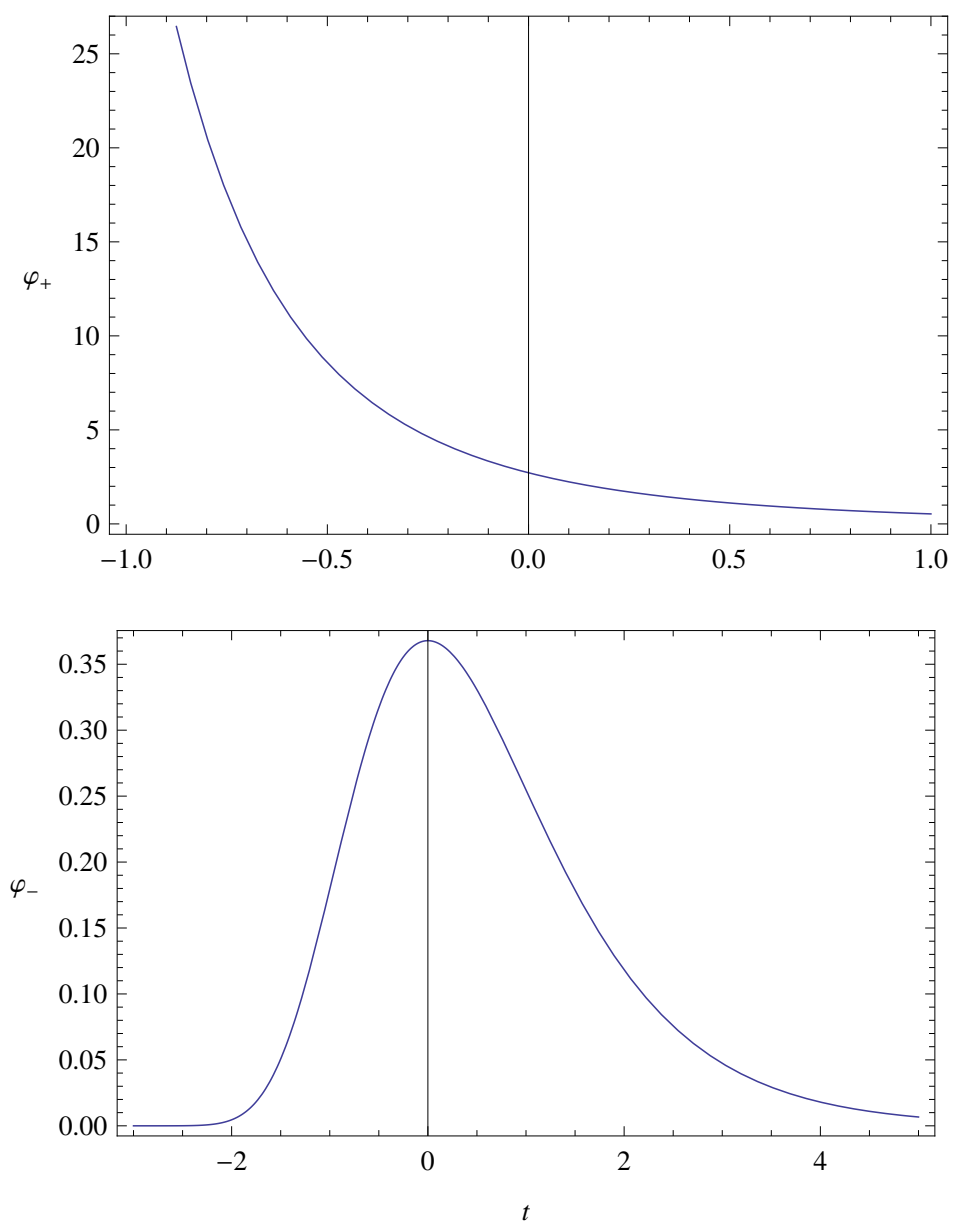

Figure 2: de Sitter $\mathrm{K}=-1$ solutions $\varphi_{ \pm}(t)$ (eq. (3.51)) with $D=4$.

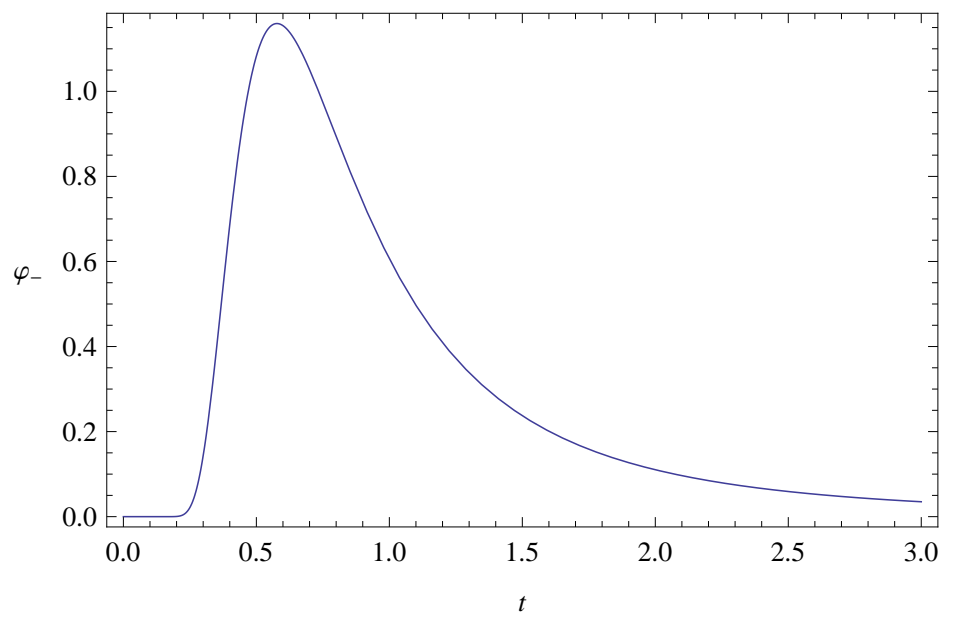

Figure 3: Power-law $\mathrm{K}=-1$ solution $\varphi_{-}(t)$ (eq. (3.53)) with $D=4$ and $p=3$.

$\varphi_{+}(t)$ has its maximum at $t<0$. This corresponds to a solution with a different initial condition for the scalar field: $\varphi_{+}$rapidly reaches the (higher) inversion point 
before the bounce.

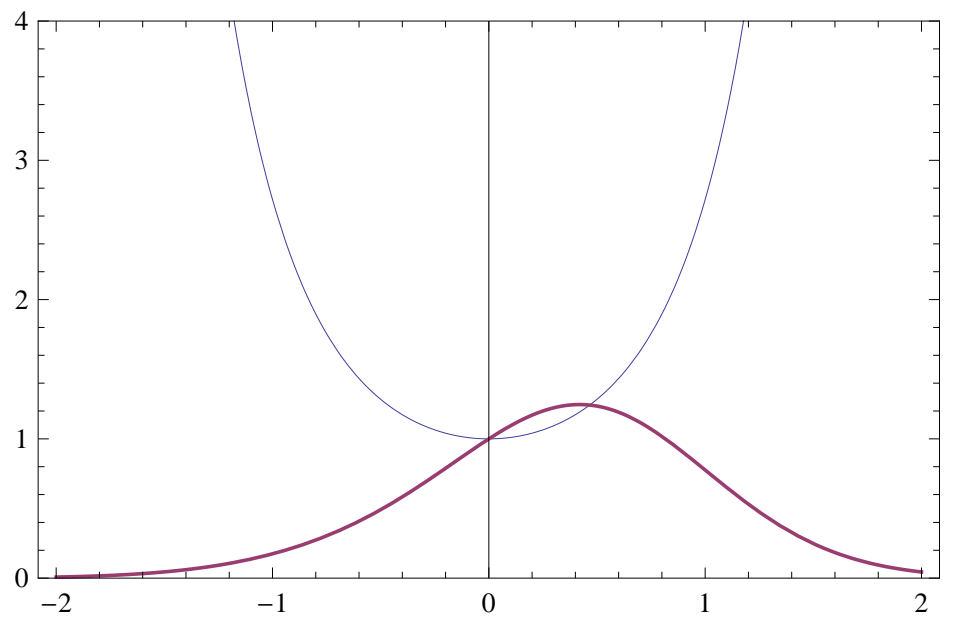

Figure 4: Asymmetric bouncing solution given by eqs. (3.50) and (3.54), with $D=4$ and $H_{0}=2$. Thin line: scale factor $a(t)$; thick line: scalar profile $\varphi_{-}(t)$.

Still in an open universe, we sketch some other solutions in four dimensions which well illustrate the exotic properties which can emerge in a non-local conformal setting. The first case is periodic in time, so we restrict it to half a period with positive scale factor $\left(r_{*}<0\right)$ :

$$
\begin{aligned}
a(t) & =\sin t, \quad t \in[0, \pi], \\
\mathrm{K} & =-1, \quad R=-12, \\
\varphi(t) & =\frac{1}{1-\cos t},
\end{aligned}
$$

which is shown in the upper panel of figure 5. Despite being open, the universe recollapses onto itself while the scalar field rolls towards the global minimum $\varphi=0$. Eventually one hits a future big crunch singularity at finite times. Another solution consists in a 'Wick rotation' of the former $\left(r_{*}>0\right)$ :

$$
\begin{aligned}
& a(t)=\sinh t, \\
& \mathrm{~K}=-1, \quad R=12, \\
& \varphi(t)=\frac{1}{\cosh t-1},
\end{aligned}
$$

depicted in the bottom panel of figure 5 .

The usual stability analysis does not apply to the above solutions because $a(t)$ is factorized out of the equations of motion. Thus, all points in the phase space plane $(\dot{a}, \dot{\varphi})$ are fixed points and there are no attractors. This is a rather bizarre situation in cosmology. Typically, a solution of the background dynamics is probed by perturbing it slightly in all sectors, inclusive gravity, as done previously. Thus 

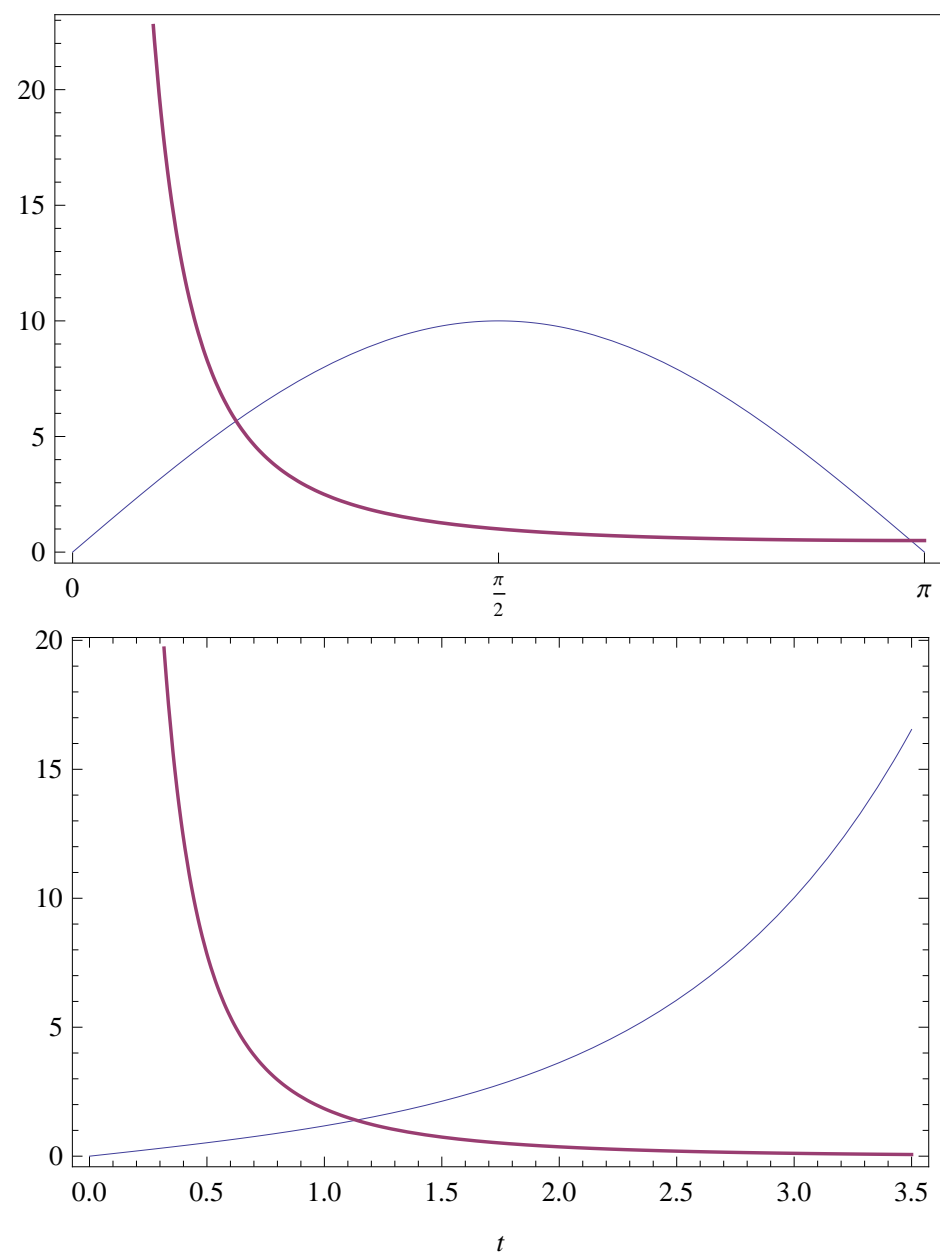

Figure 5: Upper panel: scale factor $10 a(t)$ (thin line) and scalar profile $\varphi(t)$ (thick line) for the recollapsing solution eq. (3.55). Bottom panel: scale factor $a(t)$ (thin line) and scalar profile $\varphi(t)$ (thick line) for the expanding solution eq. (3.56).

one checks whether one will hit the same solution after evolving the system from slightly different initial conditions. In the conformal system with $\alpha=-\alpha_{*}$, on the other hand, this operation is ill defined because $a(t)$ is a fixed input.

Can we conclude that the scale factor is non-dynamical and, thus, the conformal solutions of this section are not physically sensible? We argue in favour of a negative answer. Non-local models are notoriously rigid and the choice of initial conditions is much more restricted with respect to local theories with a standard Cauchy problem [1, 10, 68]. Here we see this property in action, with a further restriction of the background choice. Moreover, the scale factor factorizes out only because we have assumed trivial diffusion, eq. (3.40). A realistic stability analysis should go beyond the homogeneous level, where the solutions are no longer stationary in the diffusion sense; this would correspond to look at semi-classical (inhomogeneous) perturbations. 


\subsection{Asymptotic solutions and spontaneous symmetry breaking}

We have found exact, cosmologically non-trivial solutions which are constant along the diffusion direction but one might ask whether there exist also non-stationary diffusing profiles. This is a rather difficult question to answer because there is no systematic method to solve the equations of motion starting from a solution of the diffusion equation. In this paper we will limit ourselves to some general remarks for $f(R)=\alpha R$.

The diffusion equation transfers the non-local degrees of freedom into the extra direction [38, 41] and one can study the system locally in spacetime coordinates without risk of falling into the contradictory situations typical of non-local dynamics. In particular, it is possible to expand field profiles and equations in series and look for asymptotic solutions.

The static potential felt by the scalar particle is, according to eq. (2.8),

$$
W(\phi)=V(\phi)-\frac{1}{2} e^{r_{*} \alpha R} \phi^{2}
$$

The curvature term matches a possible quadratic term $m^{2} \phi^{2} / 2$ in $V$ and the net result is a dynamical rescaling of the squared mass, which may be even negative (tachyon). A particularly important situation is when $V$ has a minimum at the origin with a power higher than 2 (e.g., $V \sim \phi^{4}$ but it could be of any other form). In this case, the dynamical term of the potential always converts the minimum $\phi=0$ of $V$ into a local maximum of $W$, and the minima are located at $\phi_{0} \neq 0$. Classically, stable solutions should tend to these minima,

$$
\phi \stackrel{t \rightarrow \infty}{\longrightarrow} \phi_{0}
$$

Quantum mechanically, the scalar field is expected to undergo spontaneous symmetry breaking and take a non-vanishing expectation value on the vacuum, $\langle\phi\rangle_{0}=\phi_{0}$. In this case the system relaxes to pure gravity, eq. (2.10), the expectation value of the scalar field playing the role of Newton's constant. This is precisely the same kind of mechanism that happens in string theory with the dilaton field.

If the scalar field potential is to be determined a posteriori, in the presence of gravity a convenient approach is to factorize $V$ out and consider the equation of motion

$$
0=\int_{0}^{r_{*}} d s\left(D \Sigma_{00}+\Sigma\right)
$$

which can be solved for given FRW profiles. In turn, this determines the scalar field profile in the diffusion equation. Looking for asymptotically (non-vanishing) constant solutions for the matter field in eq. (3.59), one gets $D R_{00}+R=0$, that is,

$$
a^{2} \dot{H}=\frac{2 \mathrm{~K}}{D-2} \text {. }
$$


This constrains the asymptotic geometry of spacetime. When $\mathrm{K}=0$, the only solution is de Sitter. For $\mathrm{K}= \pm 1$ and $D=4$ the scale factor is, respectively, $a=(\cosh b t) / b$ and $a=(\sinh b t) / b$, where $b$ is an arbitrary constant. ${ }^{6}$ The curvature is always a positive constant, $R_{0}=12 b^{2}$. In the limit $b \rightarrow 0$, the $\mathrm{K}=-1$ solution reduces to the linear case $a=t$. This is the only nontrivial solution of eq. (3.60) with asymptotically vanishing curvature $R_{0}$. Whatever the full solutions are, they can only be constructed by considering the above scale factors either as exact or asymptotic profiles.

We conclude this part by determining the relations between the constants defining the spacetime geometry $(\Lambda$ and $G)$ and those coming from matter $\left(V_{0}=V\left(\phi_{0}\right)\right.$ and $\left.\phi_{0}\right)$. This can be achieved in two independent ways, either by looking at the action eq. (2.8) or at the the equation of motion, eqs. (2.20) and (2.21). Taking the weak non-local limit of the action $\left(r_{*} \ll 1\right)$, we get the standard Einstein-Hilbert action with cosmological and Newton constants given by

$$
\Lambda_{\phi}=\frac{1}{2 r_{*} \alpha}\left(\frac{2 V_{0}}{\phi_{0}^{2}}-1\right), \quad 8 \pi G_{\phi}=\frac{1}{r_{*} \alpha \phi_{0}^{2}} .
$$

By consistency, we expect eqs. (2.20) and (2.21) to reproduce the Einstein equations in vacuum with the values (3.61). To this aim, it is important to note that the asymptotic value of the scalar field depends on $r_{*}$. The potential $W$ is explicitly $r_{*}$-dependent and, as a consequence, so are its local minima.

Once again, we can appeal to the diffusion equation governing the flow in the $r_{*}$ variable. In fact,

$$
\phi\left(r-r_{*}, x\right)=\phi(r, x)-r_{*} \partial_{r} \phi(r, x)+O\left(r_{*}^{2}\right)=\left[1+r_{*} \square+r_{*} \alpha R+O\left(r_{*}^{2}\right)\right] \phi(r, x) .
$$

One could have just ignored the diffusion picture and consider a small $r_{*}$ expansion in $\phi\left(1-r_{*}, x\right)=e^{r_{*}(\square+\alpha R)} \phi(1, x)$,

$$
\phi\left(1-r_{*}, x\right)=\left[1+r_{*} \square+r_{*} \alpha R+O\left(r_{*}^{2}, \square^{2}\right)\right] \phi(1, x) .
$$

However, in the latter expression it is not clear whether we are entitled to safely take an asymptotic limit $\phi_{\text {asymp }}$ for $\phi$, as we have to know the contribution of all the derivatives of the field. On the other hand, the diffusion method regards $\phi(r, x)$ as a local field with two arguments, and the small $r_{*}$ expansion is a genuine local expansion. In other words, the diffusion picture justifies manipulations of asymptotic solutions in the non-local model.

Taking the asymptotic limit $x \rightarrow \infty$ of eq. (3.62), we get

$$
\lim _{x \rightarrow \infty} \phi\left(1-r_{*}, x\right)=\left[1+r_{*} \alpha R_{0}+O\left(r_{*}^{2}\right)\right] \phi_{0} .
$$

\footnotetext{
${ }^{6}$ Clearly, the symmetry breaking argument does not apply to the corresponding exact stationary solutions with quadratic potential, eqs. (3.21), (3.48) and (3.56)).
} 
Performing the asymptotic limit in the equations of motion (2.20) and (2.21), expanding in $r_{*}$ up to the first order, and taking eq. (3.64) into account one easily gets the standard Einstein equations in vacuum, with Newton and cosmological constants as in eq. (3.61).

Another interesting avenue to explore for non-stationary cosmological solutions is the conformal case $\alpha=-\alpha_{*}$. Then, the diffusion equation becomes

$$
\bar{\phi}^{\prime \prime}+\operatorname{sgn}(\mathrm{K}) \gamma^{2} \bar{\phi}-a^{3-\frac{D}{2}} \partial_{r} \bar{\phi}=0 .
$$

Interestingly, in six dimensions this is the usual homogeneous diffusion equation in conformal time, of which we know the solutions. For example, on a de Sitter background the Gaussian profile $\bar{\phi}(r, \tau)$ becomes an asymmetric kink in synchronous time, while for the power-law expansion it is a very flat lump. One should check, however, if the profiles $\phi$ solve, even asymptotically, the Einstein equations for a given potential. This is not guaranteed. In fact, unlike the case of stationary solutions the system is not trivialized to Minkowski. The reason is simple. Let

$$
\phi_{n} \equiv\left(\square-\alpha_{*} R\right)^{n} \phi .
$$

The field $\phi_{n}$ is conformal with weight $a^{-2 n}$ when $n=(D / 2-1) / 2$. Hence, none of the $\phi_{n}$ is conformal (except possibly one if $D=6+2 k, k \in \mathbb{N}$ ) and the object

$$
e^{\square-\alpha_{*} R} \phi=\sum_{n=0}^{\infty} \frac{\phi_{n}}{n !}
$$

contains an infinite number of $a$ factors, which survive in $\Sigma_{\mu \nu}$.

\section{String-like action and cosmology}

The model studied so-far is based on a gravity extension of a $p$-adic like scalar field action, which has a trivial local limit $r_{*} \rightarrow 0$ (no dynamical degrees of freedom). We can now make a slight but crucial modification of the kinetic operator $e^{\mathcal{K}}$ into a transcendental expression of the form $e^{\mathcal{K}} \mathcal{K}$ :

$$
S=\int d^{D} x \sqrt{-g}\left[\frac{1}{2} \phi e^{r_{*}[\square+f(R)]} \tilde{\phi}-V(\phi)\right],
$$

where

$$
\tilde{\phi} \equiv[\square+f(R)] \phi .
$$

The linear case $f(R)=\alpha R$ is important for several reasons already illustrated in [25]. First, when $r_{*}<0$ one formally recovers the effective spacetime action of the tachyon and the graviton in closed SFT. When gravity is switched off, eq. (4.1) reads

$$
S \sim \int d^{D} x\left[\frac{1}{2} \phi e^{-\left|r_{*}\right| \square} \square \phi-V(\phi)\right],
$$


where $\phi$ is (up to a $\phi \tilde{\phi}$ negative mass term) the tachyon field 'dressed' with an exponential operator (see, e.g., 40] for details) and $V$ is a polynomial potential. On the other hand, when $\phi=1$ we get

$$
S \sim \int d^{D} x \sqrt{-g}\left[e^{-\left|r_{*}\right|(\square+\alpha R)} R-2 \Lambda\right]
$$

This is an effective non-local gravitational action in terms of Riemann invariants. In closed SFT we do not have any such thing because the effective level-truncated action for $g_{\mu \nu}$ is obtained in a non-diffeomorphism-invariant form 45, 56. ${ }^{7}$ However, by expanding eq. (4.4) at linear order around Minkowski spacetime, one indeed gets the correct propagator $\tilde{G}(k)$ in momentum space for the graviton, as shown in [25]:

$$
\tilde{G}(k) \sim-\frac{e^{-\left|r_{*}\right| k^{2}}}{k^{2}} .
$$

This propagator is ghost free and realizes asymptotic safety at large momenta, two properties expected in a ultraviolet-finite non-perturbative theory of quantum gravity [4, 23, 25]. Therefore, eq. (4.1) is interesting both as a toy model for the spacetime effective dynamics of gravity in closed SFT and as a non-perturbative Ansatz for the gravitational action at high energies/large curvature, which can play a major role during the very early universe.

From eq. (4.1), the scalar equation of motion $\delta S / \delta \phi=0$ is

$$
\tilde{\phi}\left(1-r_{*}, x\right)=V^{\prime}[\phi(1, x)]
$$

while the Einstein equations read

$$
0=-g_{\mu \nu}\left\{\frac{1}{2} \phi(1) \tilde{\phi}\left(1-r_{*}\right)-V[\phi(1)]\right\}+\Sigma_{\mu \nu}(0)+\int_{0}^{r_{*}} d s \tilde{\Sigma}_{\mu \nu}(s)
$$

where $\tilde{\Sigma}_{\mu \nu}$ is $\Sigma_{\mu \nu}$ with $\phi\left(1-r_{*}+s\right)$ replaced by $\tilde{\phi}\left(1-r_{*}+s\right)$ (or the symmetrized expression). With obvious notation, the trace equation is

$$
0=-D\left\{\frac{1}{2} \phi(1) \tilde{\phi}\left(1-r_{*}\right)-V[\phi(1)]\right\}+\Sigma(0)+\int_{0}^{r_{*}} d s \tilde{\Sigma}(s) .
$$

The equations of motion could have been obtained also by replacing $V \rightarrow r_{*} V$ in the equations of the $p$-adic-like case, eqs. (2.19) and (2.20), and differentiating with respect to $r_{*}$. One notices, in fact, that $\partial_{r_{*}} \phi\left(1-r_{*}+s\right)=\tilde{\phi}\left(1-r_{*}+s\right)$ and that the contribution of the upper extremum of the integral, $\Sigma_{\mu \nu}\left(r_{*}\right)$, is equal to $\Sigma_{\mu \nu}(0)$ upon symmetrization (i.e., splitting $\int \Sigma$ in two and replacing $s \rightarrow r_{*}-s$ in one of the pieces).

\footnotetext{
${ }^{7}$ Nonetheless, diffeomorphisms are still part of the symmetry group of the theory [45].
} 
To get stationary solutions with $f(R)=\alpha R$, it is necessary to reinstate the normalization constant $\beta$ in the diffusion equation,

$$
\tilde{\varphi}+\beta \varphi=0
$$

which is fixed by eq. (4.6):

$$
V=\frac{m^{2}}{2} e^{2 \beta} \varphi^{2}, \quad \beta=-m^{2} e^{\beta r_{*}}
$$

The equations of motion are still $\Sigma_{\mu \nu}=0$ but with $0 \neq \beta \neq 1$. The Friedmann equations read

$$
\begin{aligned}
& 0=\left[\frac{\mathrm{K}}{a^{2}}+\left(\frac{D}{2}-1\right) H^{2}+\frac{\beta}{2 \alpha(D-1)}\right] \varphi^{2}-\frac{1}{2 \alpha(D-1)} \dot{\varphi}^{2}+2 H \dot{\varphi} \varphi \\
& 0=[4 \alpha(D-1)+D-2]\left(\dot{\varphi}^{2}+\alpha R \varphi^{2}\right)+[4 \alpha(D-1)+D] \beta \varphi^{2}
\end{aligned}
$$

The requirement $\beta \neq 0$ is very stringent, $\alpha \neq-\alpha_{*}$ and the only exact solution in common with the ' $p$-adic' case is the flat $(\mathrm{K}=0)$ de Sitter profile $(3.20)$ and $(3.21)$ with

$$
\begin{aligned}
\beta & =-\frac{\alpha(4 D \alpha+D-1)[4 \alpha(D-1)+D-2]}{(4 \alpha+1)^{2}} H^{2}, \\
\varphi(t) & =\exp \left(\frac{2 \alpha H}{4 \alpha+1} t\right)
\end{aligned}
$$

The solution is stable only for certain values of $\alpha$. In fact, the eigenvalues of the characteristic equation for the linearized system (3.25) are

$$
\lambda_{1}=\left(\frac{1}{4 \alpha+1}-D\right) H, \quad \lambda_{2}=\frac{2 \alpha H}{4 \alpha+1} .
$$

The solution is stable for

$$
-\frac{D-1}{4 D}<\alpha<0
$$

\section{Discussion}

In this paper we have constructed and solved, on cosmological backgrounds, an effective non-local model of gravity non-minimally coupled with a scalar field. The actions (2.8) and (4.1) are non-perturbative both in the order of curvature invariants,

$$
e^{R} \sim 1+R+\frac{1}{2} R^{2}+\ldots
$$

and in the number of derivatives acting on the metric,

$$
e^{\square} R \sim R+\square R+\frac{1}{2} \square^{2} R+\ldots
$$


Non-local cosmology is radically different from higher-order cosmological models of $f(R)$, Gauss-Bonnet or $f$ (Gauss-Bonnet) gravity. This is because truncation of a non-local model in spacetime derivatives produces an order $n$ Ostrogradski-like problem with altogether different physical properties. The well-known fact that theories with an infinite number of derivatives are not the large $n$ limit of finite-order actions has been also invoked to question the relevance of higher-order cosmologies in the early universe [69]. Rather than a finite-order truncation of the gravitational action, near the big bang curvature effects should be consistently taken into account only within a fully non-perturbative framework in the sense of the left-hand side of eqs. (5.1) and (5.2). The right-hand side of eq. (5.2) might not be even well-defined on a general non-local solution of the system [10]. The diffusion equation method allows one to deal with the full non-local operators and bypass the problems of a series expansion.

The diffusion structure we have explored is asymmetric in the gravity and scalar sector, in fact the former does not diffuse at all. The only solutions we have been able to find do not diffuse even in the matter sector (more precisely, they are stationary along the diffusion flow), but in general a non-stationary diffusion structure is necessary to solve the system with a self-interacting (higher-order potential) scalar field. For the purpose of finding analytic solutions, this should exclude the a priori assumption that, preferring a 'symmetric' formulation of the model, also the scalar sector does not diffuse. In this case, geometry through the curvature term $f(R)$ would replace diffusion along $r$. Therefore, the theory of diffusion associated with non-local actions would be simply defined differently: Diffusion always takes place through geometry, but in the case of trivial geometry (Minkowski background), this is realized by an auxiliary higher-dimensional structure. If this was really the case, however, it would be probably difficult to find analytic or semi-analytic solutions with non-linear self-interaction (non-quadratic $V$ ).

The actions we have studied are structurally similar to the one advanced in 25] for the following reason. On one hand, the proposal of [25] aimed at an ultraviolet finite action for quantum gravity which would address the big bang singularity problem. On the other hand, we wanted an action which would be non-local in both matter and gravity sectors and be endowed with a diffusion structure allowing one to reduce the dynamics to a set of local equations with both a second-order differential structure (in spacetime) and an algebraic structure (in the diffusion direction [10, 38, 40]). These questions, however, are implicitly related: ghost and asymptotic freedom are determined by the specific choice of pseudo-differential operators, in this case one with a natural diffusion structure. So diffusion and good ultraviolet properties are tied together, as expected in string field theory [11.

There is, anyway, a caveat in this comparison. Not only we stressed the importance of solving a fully non-local action with both gravity and matter cosmological non-trivial profiles, but in doing so it was also shown how these profiles can differ, 
even considerably, with respect to local scenarios. ${ }^{8}$ The simplest cosmological profiles (de Sitter and power law) are exact solutions of the non-local dynamics. There are a couple of remarkable facts associated with that. First, we needed only to look at stationary solutions along the diffusion flow. Second, contrary to standard general relativity these profiles correspond to exact dynamics even when the intrinsic curvature $\mathrm{K}$ is negative definite. In particular, de Sitter is an exact solution for a non-constant scalar field profile, also in an open universe. When the non-local operators are chosen to be conformal, for models with $f(R)=-\alpha_{*} R$ we have found the general solution for any flat or open FRW background, embodied by eq. (3.42). Within this class there are solutions without big bang singularity, but there also exist an infinite number of solutions with big bang. Therefore we incline not to link non-singular solutions with the ultraviolet structure of the non-local action.

At any rate, the space of solutions is likely to be much larger than the portion we have explored here. All our exact solutions have a quadratic potential. Highly nonlinear equations of motion are of great interest, especially in string theory, but the exact solutions can give some indication of the behaviour for general potentials near a local minimum. Cosmological friction modifies the dynamics of non-local scalars with respect to Minkowski and, in particular, should drastically change the rolling of the tachyon in string field theory.

\section{Acknowledgments}

Open Access. This article is distributed under the terms of the Creative Commons Attribution Noncommercial License which permits any noncommercial use, distribution, and reproduction in any medium, provided the original author(s) and source are credited.

\section{A. de Sitter and power-law solutions in standard local cos- mology}

Consider a $D$-dimensional universe filled only with a scalar field with potential $V$ and $\Lambda=0$. The standard Friedmann and continuity equations are

$$
\begin{aligned}
& \left(\frac{D}{2}-1\right) H^{2}=\frac{\kappa^{2}}{D-1}\left(\frac{\dot{\phi}^{2}}{2}+V\right)-\frac{\mathrm{K}}{a^{2}}, \\
& H^{2}+\dot{H}=\frac{\kappa^{2}}{D-1}\left[\frac{2}{D-2} V-\dot{\phi}^{2}\right], \\
& 0=\ddot{\phi}+(D-1) H \dot{\phi}+V^{\prime} .
\end{aligned}
$$

\footnotetext{
${ }^{8}$ Deviations from local cosmology is not limited to background solutions. It would be interesting to study the inflationary spectra stemming from the inhomogeneous perturbation of the Einstein equations.
} 
In de Sitter, eq. (3.20), for a flat universe the exact solution is just a cosmological constant,

$$
\phi(t)=\phi_{0}, \quad V(\phi)=\frac{(D-1)(D-2) H^{2}}{2 \kappa^{2}}, \quad \mathrm{~K}=0 .
$$

The Friedmann equations show that there is no solution if $\mathrm{K}=-1$, while there is one for a closed universe, but only in $D=4$ :

$$
\phi_{ \pm}(t)= \pm \sqrt{\frac{2}{\kappa^{2} H^{2}}} e^{-H t}, \quad \mathrm{~K}=1 .
$$

The continuity equation fixes the potential:

$$
V(\phi)=\frac{3 H^{2}}{\kappa^{2}}+H^{2} \phi^{2} .
$$

The scalar field $\phi_{ \pm}$rolls down its potential from $t=-\infty$ and climbs it again after passing the global minimum. The solution is actually unique, since cosmological equations of motion are invariant under time reversal, and it does not matter the direction of the rolling in a symmetric potential.

For a power-law expansion,

$$
a(t)=t^{p}, \quad H(t)=\frac{p}{t},
$$

one can try the profile $\phi(t)=\left(\phi_{0} / q\right) t^{q}$ in the (sum of the) Friedmann equations, but one soon finds that it must be $q=0$. This suggests to consider the limit $q \rightarrow 0$, which is a logarithmic profile:

$$
\phi(t)=\phi_{0} \ln t .
$$

This gives

$$
V(\phi)=\frac{(D-1) p-1}{2} \phi_{0}^{2} e^{-2 \phi / \phi_{0}} .
$$

If the universe is flat,

$$
\phi_{0}= \pm \sqrt{\frac{(D-2) p}{\kappa^{2}}}, \quad \mathrm{~K}=0
$$

while for a curved universe only the case $p=1$ is solution:

$$
\phi_{0}= \pm \sqrt{\frac{D-2+2 \mathrm{~K}}{\kappa^{2}}}, \quad p=1 .
$$

This solution is real if $D>2(1-\mathrm{K})$. Therefore, it is always valid for a closed universe, while for an open universe it exists only in $D>4$.

In the great majority of applications in the literature, the curvature is ignored because its contribution is washed away by inflation. However, it is interesting to note that the only curved solution in de Sitter is a closed four-dimensional universe, while for a power-law expansion both signs of the curvature are allowed but for $D \geq 5$. In $D=4$, only the closed solution is allowed. 
The non-local cosmologies described in the main body of the paper, on the other hand, only allow flat and open solutions, but without constraints on the dimensionality of spacetime. In this sense, non-local cosmologies are 'complementary' to the usual ones!

\section{References}

[1] I.Ya. Aref'eva, Nonlocal string tachyon as a model for cosmological dark energy, AIP Conf. Proc. 826 (2006) 301 [astro-ph/0410443].

[2] I.Ya. Aref'eva and L.V. Joukovskaya, Time lumps in nonlocal stringy models and cosmological applications, JHEP 10 (2005) 087 [hep-th/0504200].

[3] I.Ya. Aref'eva, A.S. Koshelev and S.Yu. Vernov, Stringy dark energy model with cold dark matter, Phys. Lett. B 628 (2005) 1 [astro-ph/0505605].

[4] G. Calcagni, Cosmological tachyon from cubic string field theory, JHEP 05 (2006) 012 [hep-th/0512259].

[5] I.Ya. Aref'eva and A.S. Koshelev, Cosmic acceleration and crossing of $w=-1$ barrier from cubic superstring field theory, JHEP 02 (2007) 041 [hep-th/0605085].

[6] I.Ya. Aref'eva and I.V. Volovich, On the null energy condition and cosmology, Theor. Math. Phys. 155 (2008) 503 [hep-th/0612098].

[7] A.S. Koshelev, Non-local SFT tachyon and cosmology JHEP 04 (2007) 029 [hep-th/0701103].

[8] I.Ya. Aref'eva, L.V. Joukovskaya and S.Yu. Vernov, Bouncing and accelerating solutions in nonlocal stringy models, JHEP 07 (2007) 087 [hep-th/0701184].

[9] I.Ya. Aref'eva and I.V. Volovich, Quantization of the Riemann zeta-function and cosmology, Int. J. Geom. Meth. Mod. Phys. 4 (2007) 881 [hep-th/0701284].

[10] G. Calcagni, M. Montobbio and G. Nardelli, Route to nonlocal cosmology, Phys. Rev. D 76 (2007) 126001 [arXiv:0705.3043].

[11] L.V. Joukovskaya, Dynamics in nonlocal cosmological models derived from string field theory, Phys. Rev. D 76 (2007) 105007 [arXiv:0707.1545].

[12] L. Joukovskaya, Rolling solution for tachyon condensation in open string field theory, arXiv:0803.3484.

[13] I.Ya. Aref'eva and A.S. Koshelev, Cosmological signature of tachyon condensation, JHEP 09 (2008) 068 [arXiv:0804.3570].

[14] L. Joukovskaya, Dynamics with infinitely many time derivatives in Friedmann-Robertson-Walker background and rolling tachyons, JHEP 02 (2009) 045 [arXiv:0807.2065]. 
[15] N.J. Nunes and D.J. Mulryne, Non-linear non-local cosmology, AIP Conf. Proc. 1115 (2009) 329 [arXiv:0810.5471].

[16] N. Barnaby, D.J. Mulryne, N.J. Nunes and P. Robinson, Dynamics and stability of light-like tachyon condensation, JHEP 03 (2009) 018 [arXiv:0811.0608].

[17] A.S. Koshelev and S.Yu. Vernov, Cosmological perturbations in SFT inspired non-local scalar field models, arXiv:0903.5176.

[18] G. Calcagni and G. Nardelli, Cosmological rolling solutions of nonlocal theories, Int. J. Mod. Phys. D 19 (2010) 329 [arXiv:0904.4245].

[19] S.Yu. Vernov, Localization of non-local cosmological models with quadratic potentials in the case of double roots, Class. Quant. Grav. 27 (2010) 035006 [arXiv:0907.0468].

[20] N. Barnaby, T. Biswas and J.M. Cline, p-adic inflation, JHEP 04 (2007) 056 [hep-th/0612230].

[21] J.E. Lidsey, Stretching the inflaton potential with kinetic energy, Phys. Rev. D 76 (2007) 043511 [hep-th/0703007].

[22] N. Barnaby and J.M. Cline, Large nongaussianity from nonlocal inflation, JCAP 07 (2007) 017 [arXiv:0704.3426].

[23] G. Calcagni and G. Nardelli, Nonlocal instantons and solitons in string models, Phys. Lett. B 669 (2008) 102 [arXiv:0802.4395].

[24] N. Barnaby and N. Kamran, Dynamics with infinitely many derivatives: variable coefficient equations, JHEP 12 (2008) 022 [arXiv:0809.4513].

[25] T. Biswas, A. Mazumdar and W. Siegel, Bouncing universes in string-inspired gravity, JCAP 03 (2006) 009 [hep-th/0508194].

[26] J. Khoury, Fading gravity and self-inflation, Phys. Rev. D 76 (2007) 123513 [hep-th/0612052].

[27] M.E. Soussa and R.P. Woodard, A nonlocal metric formulation of MOND, Class. Quant. Grav. 20 (2003) 2737 [astro-ph/0302030].

[28] S. Deser and R.P. Woodard, Nonlocal cosmology, Phys. Rev. Lett. 99 (2007) 111301 [arXiv:0706.2151].

[29] S. Nojiri and S.D. Odintsov, Modified non-local- $F(R)$ gravity as the key for the inflation and dark energy, Phys. Lett. B 659 (2008) 821 [arXiv:0708.0924].

[30] S. Jhingan, S. Nojiri, S.D. Odintsov, M. Sami, I. Thongkool and S. Zerbini, Phantom and non-phantom dark energy: the cosmological relevance of non-locally corrected gravity, Phys. Lett. B 663 (2008) 424 [arXiv:0803.2613]. 
[31] S. Capozziello, E. Elizalde, S. Nojiri and S.D. Odintsov, Accelerating cosmologies from non-local higher-derivative gravity, Phys. Lett. B 671 (2009) 193 [arXiv:0809.1535].

[32] C. Deffayet and R.P. Woodard, Reconstructing the distortion function for nonlocal cosmology, JCAP 08 (2009) 023 [arXiv:0904.0961].

[33] G. Cognola, E. Elizalde, S. Nojiri, S.D. Odintsov and S. Zerbini, One-loop effective action for non-local modified Gauss-Bonnet gravity in de Sitter space, Eur. Phys. J. C 64 (2009) 483 [arXiv:0905.0543].

[34] Ya.I. Volovich, Numerical study of nonlinear equations with infinite number of derivatives, J. Phys. A 36 (2003) 8685 [math-ph/0301028].

[35] V. Forini, G. Grignani and G. Nardelli, A new rolling tachyon solution of cubic string field theory, JHEP 03 (2005) 079 [hep-th/0502151].

[36] V.S. Vladimirov, On the equation of the p-adic open string for the scalar tachyon field, Izv. Math. 69 (2005) 487 [math-ph/0507018].

[37] G. Calcagni and G. Nardelli, Tachyon solutions in boundary and cubic string field theory, Phys. Rev. D 78 (2008) 126010 [arXiv:0708.0366].

[38] G. Calcagni, M. Montobbio and G. Nardelli, Localization of nonlocal theories, Phys. Lett. B 662 (2008) 285 [arXiv:0712.2237].

[39] D.J. Mulryne and N.J. Nunes, Diffusing non-local inflation: solving the field equations as an initial value problem, Phys. Rev. D 78 (2008) 063519 [arXiv:0805.0449].

[40] G. Calcagni and G. Nardelli, Kinks of open superstring field theory, Nucl. Phys. B 823 (2009) 234 [arXiv:0904.3744].

[41] G. Calcagni and G. Nardelli, String theory as a diffusing system, JHEP 02 (2010) 093 [arXiv:0910.2160].

[42] M. Saadi and B. Zwiebach, Closed string field theory from polyhedra, Annals Phys. 192 (1989) 213.

[43] T. Kugo, H. Kunitomo and K. Suehiro, Nonpolynomial closed string field theory, Phys. Lett. B 226 (1989) 48.

[44] T. Kugo and K. Suehiro, Nonpolynomial closed string field theory: action and its gauge invariance, Nucl. Phys. B 337 (1990) 434.

[45] V.A. Kostelecký and S. Samuel, Collective physics in the closed bosonic string, Phys. Rev. D 42 (1990) 1289.

[46] B. Zwiebach, Closed string field theory: quantum action and the B-V master equation, Nucl. Phys. B 390 (1993) 33 [hep-th/9206084]. 
[47] A. Sen and B. Zwiebach, A proof of local background independence of classical closed string field theory, Nucl. Phys. B 414 (1994) 649 [hep-th/9307088].

[48] A. Sen and B. Zwiebach, Quantum background independence of closed string field theory, Nucl. Phys. B 423 (1994) 580 [hep-th/9311009].

[49] A. Sen and B. Zwiebach, A note on gauge transformations in Batalin-Vilkovisky theory, Phys. Lett. B 320 (1994) 29 [hep-th/9309027].

[50] Y. Okawa and B. Zwiebach, Twisted tachyon condensation in closed string field theory, JHEP 03 (2004) 056 [hep-th/0403051].

[51] H. Yang and B. Zwiebach, Dilaton deformations in closed string field theory, JHEP 05 (2005) 032 [hep-th/0502161].

[52] H. Yang and B. Zwiebach, A closed string tachyon vacuum?, JHEP 09 (2005) 054 [hep-th/0506077].

[53] Y. Michishita, Field redefinitions, T-duality and solutions in closed string field theories, JHEP 09 (2006) 001 [hep-th/0602251].

[54] N. Moeller, Closed bosonic string field theory at quintic order: five-tachyon contact term and dilaton theorem, JHEP 03 (2007) 043 [hep-th/0609209].

[55] N. Moeller, Closed bosonic string field theory at quintic order. II: marginal deformations and effective potential, JHEP 09 (2007) 118 [arXiv:0705.2102].

[56] N. Moeller, A tachyon lump in closed string field theory, JHEP 09 (2008) 056 [arXiv:0804.0697].

[57] H. Yang and B. Zwiebach, Rolling closed string tachyons and the big crunch, JHEP 08 (2005) 046 [hep-th/0506076].

[58] I. Swanson, Cosmology of the closed string tachyon, Phys. Rev. D 78 (2008) 066020 [arXiv:0804.2262].

[59] L. Brekke and P.G.O. Freund, p-adic numbers in physics, Phys. Rept. 233 (1993) 1.

[60] N. Moeller and M. Schnabl, Tachyon condensation in open-closed p-adic string theory, JHEP 01 (2004) 011 [hep-th/0304213].

[61] V. Vladimirov, Nonlinear equations for p-adic open, closed, and open-closed strings, Theor. Math. Phys. 149 (2006) 1604 [arXiv:0705.4600].

[62] K. Ohmori, Toward open-closed string theoretical description of rolling tachyon, Phys. Rev. D 69 (2004) 026008 [hep-th/0306096].

[63] H. Yang, Stress tensors in p-adic string theory and truncated OSFT, JHEP 11 (2002) 007 [hep-th/0209197]. 
[64] E.J. Copeland, A.R. Liddle and D. Wands, Exponential potentials and cosmological scaling solutions, Phys. Rev. D 57 (1998) 4686 [gr-qc/9711068].

[65] G. Calcagni and A.R. Liddle, Stability of multi-field cosmological solutions, Phys. Rev. D 77 (2008) 023522 [arXiv:0711.3360].

[66] E.J. Copeland, M.R. Garousi, M. Sami and S. Tsujikawa, What is needed of a tachyon if it is to be the dark energy?, Phys. Rev. D 71 (2005) 043003 [hep-th/0411192].

[67] E.S. Fradkin and A.A. Tseytlin, Conformal supergravity, Phys. Rept. 119 (1985) 233.

[68] N. Moeller and B. Zwiebach, Dynamics with infinitely many time derivatives and rolling tachyons, JHEP 10 (2002) 034 [hep-th/0207107].

[69] G. Calcagni, B. de Carlos and A. De Felice, Ghost conditions for Gauss-Bonnet cosmologies, Nucl. Phys. B 752 (2006) 404 [hep-th/0604201]. 